\title{
FORMULATION OF CURCUMIN NANOSUSPENSION USING BOX-BEHNKEN DESIGN AND STUDY OF IMPACT OF DRYING TECHNIQUES ON ITS POWDER CHARACTERISTICS
}

\section{JASMINE KAUR, PALAK BAWA, SARVI YADAV RAJESH, PARTH SHARMA, DEEPAK GHAI, JIVAN JYOTI, SANANDA SOM, SOUVIK MOHANTA, HARISH RATHEE, ADIL HUSSAIN MALIK, SACHIN KUMAR SINGH*, BIMLESH KUMAR, MONICA GULATI, NARENDRA KUMAR PANDEY, VARUN GARG, ANKIT KUMAR YADAV, RAKESH NARANG}

Department of Quality Assurance, School of Pharmaceutical Sciences, Lovely Professional University, Phagwara, Punjab, India. Email: singhsachin23@gmail.com

Received: 14 July 2017, Revised and Accepted: 25 July 2017

ABSTRACT

Objective: The objective of this study was to formulate curcumin nanosuspension (NS) using Box-Behnken design (BBD) and solvent-antisolvent technique to overcome the challenges related to its poor dissolution rate.

Methods: Sodium lauryl sulfate (SLS) and poly vinyl pyrrolidone K-60 (PVPK-60) have been used as a surfactant and polymer, respectively, to stabilize the NS. Ethanol was used as solvent to dissolve curcumin and water was used as antisolvent. The study revealed that SLS to curcumin ratio, PVPK-60 to curcumin ratio, solvent to antisolvent ratio and speed of mixing were the critical parameters that affected particle size and zeta potential of the formulation. Hence, based on Box- BBD, 25 formulations were prepared by varying these critical parameters. The optimized batch of CRM NS was further solidified using spray drying as well as rotary evaporation techniques to have a better insight for selection of solidification process in terms of retention of particle size, charge, flow, dissolution, and stability.

Results: About 39.47 folds decrease in particle size of raw CRM was observed after conversion into NS. Further, about 53.57 and 45.45 folds decrease in particle size was observed after spray drying and rotary evaporation. Both the dried nanoparticles have shown comparatively higher solubility, powder flow, and dissolution rate as that of raw CRM. Powder X-ray diffraction study revealed the formation of amorphous nanoparticles. Accelerated stability study revealed that nanoparticles dried by spray drying were able to retain the properties such as particle size, flow, and dissolution rate as compared to rotary evaporated powders.

Conclusion: It can be concluded that spray drying technique could offer many advantages while loading CRM nanoparticles into tablets for their oral administration.

Keywords: Curcumin, Spray drying, Rotary evaporation, Box-behnken design, Dissolution, Stability studies.

(C) 2017 The Authors. Published by Innovare Academic Sciences PvtLtd. This is an open accessarticle under theCC BY license (http://creativecommons. org/licenses/by/4. 0/) DOI: http://dx.doi.org/10.22159/ajpcr.2017.v10s4.21335

\section{INTRODUCTION}

Curcumin (CRM) is a potent phytoconstituent derived from turmeric (Curcuma longa, Family Zingiberaceae) [1]. It has been mainly reported for its anticancer properties and found to be beneficial as antibacterial, antifungal, antiamoebic, antioxidant, antidiabetic, anti-HIV and in neuro-generative and respiratory diseases [2-9]. Despite having an array of medicinal properties, there are certain challenges with its physicochemical properties such as low aqueous solubility $(3.12 \mathrm{~g} / \mathrm{L}$, $25^{\circ} \mathrm{C}$ ), degradation at intestinal $\mathrm{pH}$, fast hepatic metabolism and elimination, which reduces its bioavailability [10-12].

Poor aqueous solubility of CRM is a major challenge for its oral administration. Various methods have been reported earlier to enhance its solubility and dissolution rate. These include the formulation of solid dispersion, liposomes, complexation with phospholipids, and cyclodextrin [13-15]. Conjugation of bioavailability enhancers such as piperine, Bio-curcumin-95 (BCM-95) has been used as alternative strategies to enhance its bioavailability [16-19]. In the past decade, nanotechnology has emerged as a unique approach that has found enormous applications in delivering lipophilic drugs with better bioavailability $[10,20]$. In this approach, submicron particles are formed which get stabilized by use of steric and electrostatic stabilizers [21]. Among nanotechnology approaches that are used for the formulation of lipophilic drugs, nanosuspension (NS) has been reported as one of the most promising techniques to improve the dissolution rate limited bioavailability of such drugs [22]. In general, bottom up and top down techniques are used to prepare NS [22,23]. In bottom up process, drug is added in solvent that dissolves it completely and then added to antisolvent by aid of stirring to form precipitates. Different techniques such as solvent-antisolvent method and super critical fluid process fall under bottom up processes. In top down process, drug is suspended in the solvent and particle size reduction was carried out by ball milling, media milling, high-pressure homogenization, and microfluidization $[22,23]$.

It is important to note that formulation of NS is greatly affected by technique used to prepare it, most importantly their physicochemical stability [24]. Top down method creates physical and chemical instability like crystal deformities and break down of heat labile drugs due to the generation of heat during milling process [24]. Whereas, by using bottom up technique, morphology and crystallinity of particles could be maintained. Supercritical fluid process under bottom up technique has been used to prepare NS on large scale [22,23]; however, it suffers from certain limitations such as operation at high pressure, temperature, and requirement of fine designed nozzles [23,24]. Alternatively, solventantisolvent method has been extensively reported to prepare NS due to its simplicity and ease of scale up. Solvent-antisolvent method is one of the simplest method which can be employed for small scale batches in laboratory and does not require already micronized particles.

Reduced particle size may lead to create high total surface energy which may lead to instability and particle agglomeration also known 
as Ostwald ripening, thereby lead to inconsistent dosing. Therefore, conversion of liquid suspension into dry powder is the best option for commercialization of NS to improve their physicochemical instability. Technologies used for drying of NS include spray drying, freeze drying, and rotary evaporation. Among them, freeze drying and spray drying are two most widely used techniques. Freeze drying can be used for drying of aqueous suspension only. Suspensions containing organic/ alkaline/acidic solvent are cumbersome to be dried using freeze dryer as it damages the instrument. Hence, critical care is required. Freeze drying may also modify the nature of active pharmaceutical ingredient, i.e., it may affect crystalline nature of drugs like generation of amorphous form or may cause some polymorphic changes [25]. Moreover, freeze drying is a time-consuming process as compared to rotary evaporation and spray drying. In rotary evaporation, all type of drugs can be dried, however aqueous dispersions take longer time to get dried. On the other hand, organic dispersions can be dried in a short time [26]. Spray drying is most desirable technique used so far for drying of NS. There have seen many reports which support spray drying technique due to its various advantages that are hard to be achieved by other techniques [26]. Spray drying process directly atomizes the drug in a hot gas current to obtain powder $[10,20]$. The spherical particles obtained have better flow property than conventional methods used for drying [27]. Furthermore, this process gives the powdered NS in very less time and can be used for both aqueous and organic dispersions. Moreover, thermolabile compounds such as proteins and peptides can also be dried using this technique [28]. Thus, spray drying has better scope for scale up.

In view of the above facts, this study is aimed toward preparation of NS of CRM using solvent-antisolvent addition technique to increase its dissolution rate. Box-Behnken design (BBD) has been used to optimize the formulation variables that could affect the particle size and zeta potential of the NS. Moreover, a comparative study of two drying techniques, i.e., spray drying and rotary evaporation has been carried out to investigate their effect on particle size, flow, compression properties, dissolution behavior as well stability of NS. The overall objective of the study was to prepare NS of curcumin and selection of best drying technique that could provide better micromeritic and stability properties to formulate the drug into a tablet for oral administration. The formed tablet could be able to improve the dissolution rate and release the drug completely within first $1 \mathrm{hr}$ of its oral intake in the gastric fluid itself; hence, the degradation of CRM at intestinal $\mathrm{pH}$ could also be avoided.

\section{MATERIALS AND METHODS}

\section{Materials}

Curcumin, dicalcium phosphate; hydroxyl propyl methyl cellulose (HPMC); poly vinyl pyrrolidone (PVP-K30 and 60) were purchased from Central drug house Pvt. Ltd. Mumbai, India. Sodium lauryl sulfate (SLS) was purchased from BB chemicals, Mumbai, India. Polyethylene glycol (PEG) 1500, 4000 and 6000, sodium hydroxide, potassium dihydrogen orthophosphate, lactose, sodium starch glycolate (SSG), methanol were purchased from Loba Chemie Pvt. Ltd., Mumbai, India. Magnesium stearate was procured from SD Fine Chemicals Ltd. Mumbai, India, and HPMC 6 cps as well as microcrystalline cellulose (MCC) PH102 was gifted by Colorcon, Mumbai, India. Ethanol was purchased from Changshu Yangyuan Chemical, China. Pluronic F68, F-127 were purchased from BASF (Ludwigshafen, Germany). Millipore water was collected from Bio-Age equipment Ltd. Mohali, Punjab, India). Ultraviolet (UV)-visible spectrophotometer double beam (UV-1800, Shimadzu Co. Ltd., Japan), hot air oven (Cadmach drying oven, Cadmach Machinery Ltd. Ahmadabad, India), rotary evaporator (IKA RV8, ESCY Enterprises, India), spray dryer (Spray mate, JISL, India), ultrasonicator (ATS.02, Athena, India), mechanical stirrer (Remi, Vasai; Mumbai, India), weighing balance (AX 200, Shimadzu, Japan), pH meter (LT-10, LABRONICS, India), tablet compression machine (Trover, Pharma Mach, India), and dissolution apparatus (LAB DS 8000, Lab India, India) were used in the study.

\section{Selection of solubilizers}

To select best possible polymer and surfactant to prepare NS of CRM, solubility study was performed using various surfactants (SLS, PEG $1500,4000,6000)$, Pluronics (F-68 and F-127) and polymers (PVP-K30 and 60, HPMC 6 cps) at different concentrations such as $0.5,1.5,2 \% \mathrm{w} / \mathrm{v}$ at room temperature. As per the concentration required, surfactants and polymers were weighed and transferred to $500 \mathrm{~mL}$ volumetric flask containing $250 \mathrm{~mL}$ distilled water and dissolved. This was followed by addition of CRM (50 mg) and stirred on magnetic stirrer for $48 \mathrm{hrs}$. Each solution was filtered using Whatman filter paper and analyzed at $420 \mathrm{~nm}$ using UV spectrophotometer.

\section{Selection of solvent and antisolvent}

Selection of optimal solvent and antisolvent could aid the formulation of NS. The solubility of CRM was carried out in acetone, methanol, and ethanol to select suitable solvent with maximum solubility as well as drug loading providing super-saturated solution that would aid in precipitation rapidly. Drug was completely soluble in ethanol; thus, it was selected as solvent. Since CRM is a lipophilic drug with poor aqueous solubility and solvent is completely miscible with water; hence, water was selected as antisolvent for nucleation and precipitation.

\section{Design of experiments (DoE)}

A total 25 different batches of NS containing CRM were formulated by liquid antisolvent precipitation method using BBD by varying the polymer to drug ratio, surfactant to drug ratio, solvent/antisolvent ratio, and speed of mixing. These factors were selected on the basis of initial trials which revealed that these affect the particle size d (90) [PSD $d(90)]$ and zeta potential of NS (results not shown). These factors were operated at three levels $(+1,0,-1)$, keeping the concentration of drug, type of polymer, type of surfactant, and time of mixing constant for all the experiments. Design-expert 10.0.3 software was used to conduct the study and experiments were run in random order to increase the predictability of the model. Table 1 shows the independent factors and their design level used in this study.

\section{Preparation of CRM NS using antisolvent precipitation technique}

CRM was dissolved in ethanol and injected by using syringe on to the tip of the antisolvent water containing specific concentration (as per DoE) of PVPK-60 and SLS with stirring. Drug precipitation took place immediately on mixing and formed a suspension with transparent light yellowish appearance. A total 25 batches of CRM-NS were prepared as per DoE (Table 2).

\section{Drying of NS}

Method I: Rotary evaporation

The optimized batch of NS was dried using rotary evaporator (IKA RV8, ESCY Enterprises, India) at a temperature of $110^{\circ} \mathrm{C}$ and reduced pressure of 5 bar and rotation speed of $100 \mathrm{rpm}$. The obtained powders were denoted as CRM-NS (RD).

Table 1: Variables for Box-Behnken study

\begin{tabular}{|c|c|c|c|}
\hline \multicolumn{2}{|l|}{ Independent factors } & \multicolumn{2}{|c|}{ Design level } \\
\hline Uncoded & Coded & Uncoded & Coded \\
\hline \multirow[t]{3}{*}{ SLS to CRM ratio (w/w) } & $\mathrm{A}(\mathrm{X} 1)$ & $0.2-1.0$ & -1 \\
\hline & & $0.4-1.0$ & 0 \\
\hline & & $0.6-1.0$ & +1 \\
\hline \multirow[t]{3}{*}{ PVP K60 to CRM ratio (w/w) } & $\mathrm{B}(\mathrm{X} 2)$ & $0.15-1.0$ & -1 \\
\hline & & $0.30-1.0$ & 0 \\
\hline & & $0.45-1.0$ & +1 \\
\hline \multirow[t]{3}{*}{ Solvent/antisolvent ratio $(\mathrm{mL})$} & C (X3) & 0.1 & -1 \\
\hline & & 0.2 & 0 \\
\hline & & 0.3 & +1 \\
\hline \multirow[t]{3}{*}{ Speed of mixer (rpm) } & $\mathrm{D}(\mathrm{X} 4)$ & 1000 & -1 \\
\hline & & 2500 & 0 \\
\hline & & 4000 & +1 \\
\hline
\end{tabular}


Table 2: Factor level and response data for BBD study

\begin{tabular}{|c|c|c|c|c|c|c|}
\hline Runs & $\begin{array}{l}\text { Factor - } 1 \mathrm{~A} . \\
\text { SLS to CRM } \\
\text { ratio }(\mathrm{X} 1)(\mathrm{g})\end{array}$ & $\begin{array}{l}\text { Factor - } 2 \text { B. } \\
\text { PVP K60 to } \\
\text { CRM ratio (X2) (g) }\end{array}$ & $\begin{array}{l}\text { Factor - } 3 \text { C. Solvent/ } \\
\text { antisolvent ratio }(\mathrm{X} 3)(\mathrm{mL})\end{array}$ & $\begin{array}{l}\text { Factor - } 4 \text { D. } \\
\text { Speed of mixing } \\
\text { (X4) (rpm) }\end{array}$ & $\begin{array}{l}\text { Response - } 1 \text { Particle } \\
\text { size d (90) [PSD d } \\
(90)](Y 1)(n m)\end{array}$ & $\begin{array}{l}\text { Response }-2 \\
\text { Zeta potential } \\
(\mathrm{mV})\end{array}$ \\
\hline 1 & 0.2 & 0.15 & 0.2 & 2500 & 732 & -23 \\
\hline 2 & 0.6 & 0.15 & 0.2 & 2500 & 628 & -30 \\
\hline 3 & 0.2 & 0.45 & 0.2 & 2500 & 692 & -16 \\
\hline 4 & 0.6 & 0.45 & 0.2 & 2500 & 676 & -28 \\
\hline 5 & 0.4 & 0.3 & 0.1 & 1000 & 802 & -18 \\
\hline 6 & 0.4 & 0.3 & 0.3 & 1000 & 766 & -17 \\
\hline 7 & 0.4 & 0.3 & 0.1 & 4000 & 718 & -28 \\
\hline 8 & 0.4 & 0.3 & 0.3 & 4000 & 686 & -27 \\
\hline 9 & 0.2 & 0.3 & 0.2 & 1000 & 850 & -14 \\
\hline 10 & 0.6 & 0.3 & 0.2 & 1000 & 806 & -29 \\
\hline 11 & 0.2 & 0.3 & 0.2 & 4000 & 712 & -20 \\
\hline 12 & 0.6 & 0.3 & 0.2 & 4000 & 602 & -32 \\
\hline 13 & 0.4 & 0.15 & 0.1 & 2500 & 734 & -24 \\
\hline 14 & 0.4 & 0.45 & 0.1 & 2500 & 686 & -20 \\
\hline 15 & 0.4 & 0.15 & 0.3 & 2500 & 751 & -25 \\
\hline 16 & 0.4 & 0.45 & 0.3 & 2500 & 717 & -16 \\
\hline 17 & 0.2 & 0.3 & 0.1 & 2500 & 812 & -19 \\
\hline 18 & 0.6 & 0.3 & 0.1 & 2500 & 778 & -23 \\
\hline 19 & 0.2 & 0.3 & 0.3 & 2500 & 796 & -13 \\
\hline 20 & 0.6 & 0.3 & 0.3 & 2500 & 682 & -27 \\
\hline 21 & 0.4 & 0.15 & 0.2 & 1000 & 714 & -20 \\
\hline 22 & 0.4 & 0.45 & 0.2 & 1000 & 816 & -23 \\
\hline 23 & 0.4 & 0.15 & 0.2 & 4000 & 657 & -28 \\
\hline 24 & 0.4 & 0.45 & 0.2 & 4000 & 663 & -27 \\
\hline 25 & 0.4 & 0.3 & 0.2 & 2500 & 731 & -25 \\
\hline
\end{tabular}

\section{Method II: Spray drying}

In the second method, spray drying was used to dry NS under the following set of conditions: Inlet temperature: $110^{\circ} \mathrm{C}$, outlet temperature: $55^{\circ} \mathrm{C}$, aspiration air flow rate of $1400 \mathrm{rpm}$, and feed pump speed of $16 \mathrm{rpm}$. The product obtained was packed immediately in air tight container for further study. The obtained powders were denoted as CRM-NS (SD).

\section{Characterization of CRM nanoparticles \\ Calculation of percentage yield}

Percentage yield was calculated by comparing sample absorbance to standard absorbance. About 1 ppm solution of pure CRM (standard) was prepared using ethanol, and $1 \mathrm{ppm}$ of nanoparticles (sample) was prepared using water. The absorbance of both solutions was taken at $420 \mathrm{~nm}$, and percentage recovery was calculated as given in equation 1.

Percentage yield $=$ Sample absorbance $/$ standard absorbance $\times 100$

\section{Particle size and zeta potential analysis}

The particle size and zeta potential of NS were measured following the principle of photon correlation spectroscopy using Beckman Coulter (Delsa ${ }^{\mathrm{TM}}$ Nano Submicron Particle Size and Zeta Potential, BREA). Before scanning, raw CRM (CRM-R), CRM-NS (SD), and CRM-NS (RD) were reconstituted in $100 \mathrm{~mL}$ of purified water to have $0.1 \mathrm{w} / \mathrm{v}$ colloidal dispersion. CRM-NS was also subjected for analysis. Samples were measured in triplicate and mean data were recorded.

\section{Micromeritic characterization of powders}

The dried powders and powders mixed with excipients used for formulating tablet (tablet blend) were further subjected to micromeritic characterization for bulk density, tapped density, angle of repose, Carr's compressibility index, and Hausner's ratio as reported in Beg et al., 2016 [29].

\section{Solubility studies}

"To compare the enhancement in solubility of prepared nanoparticles, i.e., CRM-NS (SD) and CRM-NS (RD) with respect to raw CRM in Millipore water, CRM-R (50 mg) and its equivalent powder from CRM-NS (SD) and CRM-NS (RD) were added separately to a flask containing $200 \mathrm{ml}$ Millipore water. Flasks were shaken on a mechanical shaker for $48 \mathrm{hrs}$ at room temperature. The solutions were then filtered (cut-off $0.2 \mu \mathrm{m}$, Ministart SRP 25, Sartorius) and analyzed. The studies were performed in triplicate and mean data were recorded [30]."

\section{Powder X-ray diffraction (PXRD) analysis}

"PXRD patterns were recorded as per Kaur et al. [30]. In brief, CRM-R, CRM-NS (SD), and CRM-NS (SD) were recorded using an X-ray diffractometer (Bruker Axs, D8 Advance) with $\mathrm{Cu}$ line as the source of radiation. Standard runs using a $40-\mathrm{kV}$ voltage, a $40 \mathrm{~mA}$ current, at a scanning rate of 0.010 minutes $^{-1}$ over a $2 \theta$ range of $3-45^{\circ}$ were used [3]."

\section{Formulation of tablets}

An amount equivalent to $5 \mathrm{mg}$ of CRM was weighed from CRM-NS (SD) powder and mixed with excipients, MCC PH102 (250 mg), lactose (100 mg), dicalcium phosphate (50 mg), SSG (30 mg) and magnesium stearate $(20 \mathrm{mg})$. The blend was finally compressed into tablet by using multi punching tablet machine (Trover Phar Mach, India). In a similar way, tablets were compressed for CRM-NS (RD) and CRM-R powders.

\section{Dissolution studies}

An amount equivalent to $5 \mathrm{mg}$ was accurately weighed from CRM-NS (SD) and CRM-NS (RD) and filled separately into hard gelatin capsules. In a similar way, $5 \mathrm{mg}$ of CRM-R was also weighed and filled in hard gelatin capsule shell. All the three capsules mentioned above as well as CRM-NS (SD), CRM-NS (RD), and CRM-R tablets were subjected for dissolution studies. For capsules, the study was carried out using USP-I, basket type apparatus and for tablets study was carried out using USP-II paddle type apparatus in $900 \mathrm{~mL}$ of $0.1 \mathrm{~N}$ hydrochloric acid at $37 \pm 0.2^{\circ} \mathrm{C}$ at $100 \mathrm{rpm}$. At specified intervals of $5,10,15,30$, 45 and 60 minutes; $5 \mathrm{~mL}$ of dissolution medium was withdrawn and replaced with an equal volume of medium to maintain constant volume. Samples were filtered through a $0.25 \mu \mathrm{m}$ membrane filter and analyzed for drug release at $420 \mathrm{~nm}$ using double beam UV-visible spectrophotometer. 
Stability studies

The accelerated stability study was carried out for CRM-NS (SD), and CRM-NS (RD) powders at $40^{\circ} \mathrm{C}$ and $75 \%$ relative humidity (RH) for 6 months. The stability of nanoparticles was judged based on particle size [PSD d (90)], angle of repose, and dissolution studies. The procedure to carry out such studies has been discussed in their corresponding previous sections.

\section{Statistical analysis of data}

All the data were statistically analyzed by analysis of variance (ANOVA). Results were quoted as significant where $\mathrm{p}<0.05$. The samples were characterized by comparing dissolution profiles of fresh and accelerated samples after 6 months using similarity factor as defined by the equation 2 :

$$
\mathrm{f}_{2}=50 \log \left\{\left[1+\frac{1}{n} \sum_{\mathrm{t}=1}^{\mathrm{n}}\left(\mathrm{R}_{\mathrm{t}}-\mathrm{T}_{\mathrm{t}}\right)\right]^{-0.5} \times 100\right\}
$$

Where, $\mathrm{n}$ is number of time points at which \% drug dissolved was determined, $\mathrm{R}_{\mathrm{t}}$ the $\%$ drug dissolved of one formulation at a given time point and $\mathrm{T}$ is the $\%$ drug dissolved of the formulation to be compared at the same time point. The similarity factor fits the result between 0 and 100. An $\mathrm{f}_{2}$ above 50 indicates that the two profiles are similar [31].

\section{RESULTS AND DISCUSSION}

\section{Selection of solubilizers}

Different concentrations of surfactants and polymers were prepared and analyzed and percentage solubility was calculated. Among all the surfactants and polymers used for solubility studies, it was found that SLS and PVPK-60 have shown highest solubility, i.e., $48 \%$ and $2.13 \%$ respectively at $2 \% \mathrm{w} / \mathrm{v}$ concentration. Hence, these were selected as solubilizers. The percentage solubility of each solubilizer at four different concentrations is shown in Fig. 1.

\section{Effect of formulation and process variables on particle size and} zeta potential

To check the effect of formulation and process variables on particle size and zeta potential of CRM NS, 25 different experiments were carried out using BBD and their responses were measured (Table 2). It was observed that the obtained results were well represented by a linear function of the independent variables; hence, the first order polynomial was used for approximating the function as shown in equation 3 .

$Y=\beta+\beta_{1} X_{1}+\beta_{2} X_{2}+\beta_{3} X_{3}+\beta_{4} X_{4}+€$
Where, $€$ represents noise or error, $\mathrm{X}$ represents independent variable, $Y$ represents response, and $\beta$ represents coefficient. The lowest value for Y1 (particle size) was $602 \mathrm{~nm}$ and Y2 (Zeta potential) was -13 $\mathrm{mV}$, whereas, the highest value for Y1 (particle size) was $816 \mathrm{~nm}$ and Y2 (Zeta potential) was $-32 \mathrm{mV}$, respectively. The results of ANOVA revealed a significant effect of variables on the responses $(p<0.05)$. The results of ANOVA confirms the adequacy of the model (Table 3). It also helped to identify the significant factors that affect the responses Y1 and Y 2 of NS. The final mathematical model in terms of coded factors as determined by design expert software is shown below in equations 4 and 5 for response $\mathrm{Y} 1$ and $\mathrm{Y} 2$, respectively.

$$
\text { Y1 }[\text { PSD d (90)] = +731.00-35.17×A+2.83×B-11.00×C }-59.67 \times \mathrm{D}
$$

Y2 [zeta potential $]=+21.72+3.50 \times \mathrm{A}-2.83 \times \mathrm{B}+0.50 \times \mathrm{C}+4.33 \times \mathrm{D}$

A positive sign represents a synergistic effect, while a negative sign indicates an antagonistic effect. In case of Y1 negative coefficients of A, C and D refer to decreased particle size at higher levels of SLS to CRM ratio, solvent to antisolvent ratio and speed of mixing, respectively. Similarly, the positive coefficients of B indicated increase in particle size with increase in ratio of PVPK-60 to CRM. Whereas, for Y2 the negative coefficients of B referred to decrease in the zeta potential with increase in the concentration of PVPK-60. However, the positive coefficients of $\mathrm{A}, \mathrm{C}$ and $\mathrm{D}$ refer to increase in zeta potential with increase in surfactant concentration, solvent to antisolvent ratio and speed of mixing. It was observed that the particle size decreased as the concentration of SLS was increased and at lower concentration of PVPK-60. PVPK-60 is vinyl pyrrolidone/vinyl acetate copolymer, and is acknowledged to get adsorb on the surface of the drug particle and resist crystal growth. When two particles surrounded by an adsorbed polymer layer approach each other, there will be a local increase in polymer concentration in that region which could result in an osmotic pressure locally and may lead to an increase in total potential energy that might be responsible for prevention of agglomeration of the particles. However, a further increase in the concentration of PVPK- 60 could result in a decrease in diffusion of the solvent toward the antisolvent caused by the high viscosity of

Table 3: Summary of ANOVA for responses

\begin{tabular}{llll}
\hline Response & \multicolumn{2}{l}{ Regression parameters } & p value \\
\cline { 2 - 3 } & $\mathbf{R}^{2}$ & Fcal & \\
\hline Particle size & 0.84 & 13.37 & 0.0020 \\
Zeta potential & 0.82 & 32.11 & $<0.0001$ \\
\hline
\end{tabular}

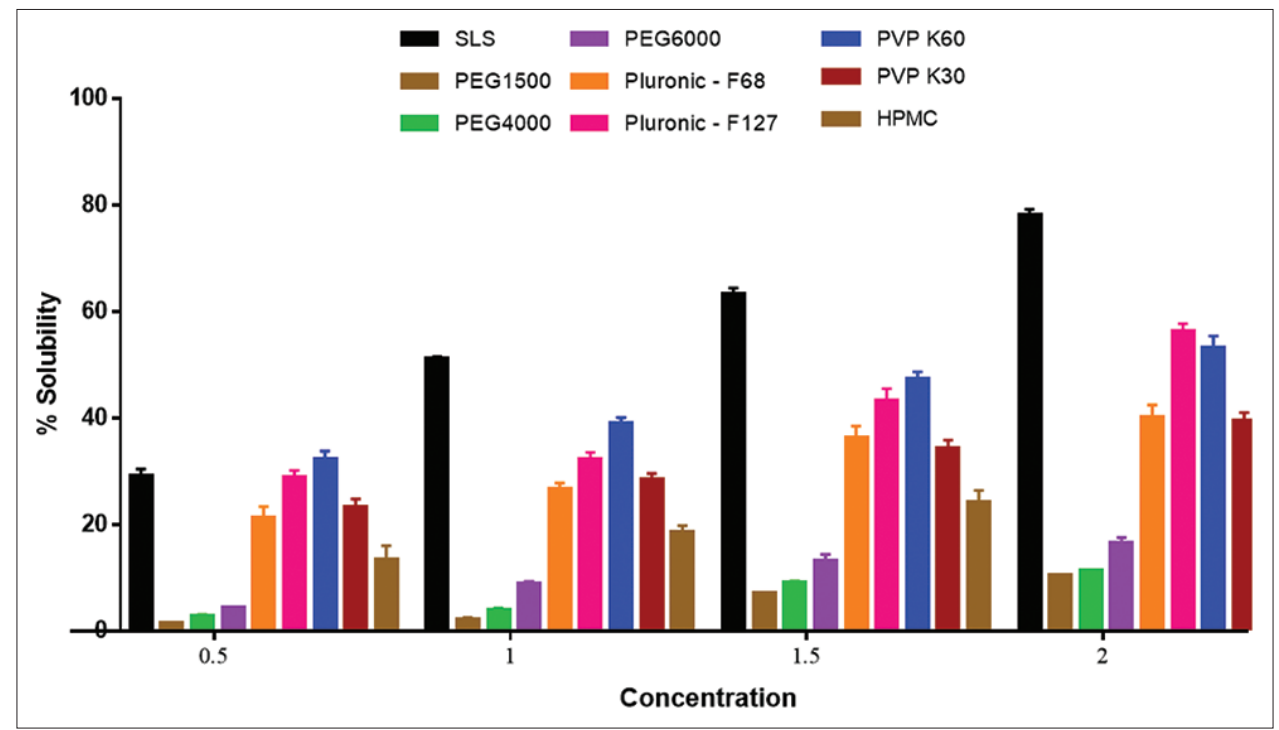

Figure 1: Solubility studies of curcumin in different solubilizers 
the solution, which in turn increases the mean particle size [32]. On the other hand, particle size was found to be decreased because of the excellent dispersion property of SLS. SLS is an anionic surfactant and its surface active property improves the wettability of the drug powder in the dispersion medium that may contribute to a reduction in mean particle size. The CRM particles did not wet adequately and particles floated on the aqueous vehicle while when SLS was employed, it increased the wettability of CRM particles and hence showed better particle size reduction [33]. With increase in solvent to antisolvent ratio, the mean particle size of CRM also increased irrespective of the fact that the concentration of surfactant was high. This could occur due to solvent mediated transformation during the crystal growth that could have led to Ostwald ripening which in turn led to increased size of CRM nanoparticles. Smaller particles were obtained at low concentration of surfactant and high speed of mixing and vice versa. It could be possibly due to generation of high energy and shear force that could have provided sufficient impact for medium to break down the precipitated drug crystals to nanometer range. Hence, it can be inferred that less amount of surfactant at high speed of mixing was sufficient for particle size reduction. Similar observations were found when particle size was significantly reduced at high speed of mixing irrespective of the concentration of PVPK-60. Moreover, the study also revealed that at lower ratio of solvent to antisolvent and high speed of mixing, the particle size was significantly reduced as compared to high ratio of solvent to antisolvent and low speed of mixing.

An increase in the zeta potential value was observed at low level of PVP (factor B) and high level of SLS (factor A). This could be due to the fact that at low level of PVP, CRM particles were not covered so densely with PVPK-60 and this allowed faster diffusion of SLS to the particle surfaces. SLS is an excellent suspending agent, adsorption of SLS on to the particles' surface leads to a high zeta potential value. When level of PVPK-60 was high, reduction in zeta potential value of NS was observed, irrespective of higher level of SLS. This could be due to shift in the plane of shear due to adsorption of PVPK-60 on CRM's surface at which the zeta potential is measured to a larger distance from the particles' surface and lowered the zeta potential. It was also observed that there was a significant difference in the zeta potential with increase or decrease in speed of mixing. At high speed of mixing an increase in zeta potential was observed because at high speed of mixing the adsorption of steric and electrostatic stabilizer was more which increases the particle mobility and zeta potential value. However, there was no much effect of solvent to antisolvent ratio on zeta potential. The polynomial equation further helped to draw 3D plots revealing the combined effect of two factors on the different responses, by keeping one factor constant in each case (Fig. 2).

Fig. 2a revealed that with increase in the ratio of SLS, the PSD d (90) got decreased, whereas, with increase in ratio of PVPK-60, PSD d (90) got increased. On the other hand, from Fig. 2b, it was understood that with the increase in SLS and S/AS ratio, PSD d (90) got decreased. Similar observations were found when factors A and D were compared (Fig. 2c). Whereas, no much change in particle size was observed with increase or decrease in factors B and C (Fig 2d). With increase in speed of mixing and PVPK-60, particle size [PSD d (90)] was found to be decreased (Fig. 2e). This could be attributed to high shear rate produced due to mixing that could have caused reduction in particle size. Fig. $2 \mathrm{f}$ represented that when effect of factors $\mathrm{C}$ and $\mathrm{D}$ was considered, there was no difference in particle size [PSD d (90)] with change in S/AS ratio, however, with increase in speed of mixing the PSD d (90) got decreased. Hence it was clear from the 3D plots that SLS ratio and speed of mixing played critical role in particle size reduction. In case of zeta potential, it was observed that it got significantly increased with increase in SLS to CRM ratio (Fig. 2g) and decreased with increase in PVPK-60 to CRM ratio (Fig. 2h) and decreased with increase in S/AS ratio (Fig. 2i). Whereas, it was found to get significantly increased in increase in speed of mixing.
Optimization of formulation and processing parameters using graphical optimization method

Optimization of NS was performed to find the levels of factors A-D which gave particle size d (90) Y1 of 500-750 nm range and Y2 in -24--31.6 $\mathrm{mV}$ range. Under this model predicted $\mathrm{Y} 1$ and $\mathrm{Y} 2$ in required range at $\mathrm{A}$, $\mathrm{B}, \mathrm{C}$ and $\mathrm{D}$ values of $0.58 \mathrm{~g}$ (ratio of surfactant to drug), $0.15 \mathrm{~g}$ (ratio of polymer to drug), 0.3 (solvent/antisolvent ratio), and $2749 \mathrm{rpm}$ (speed of mixing), respectively for a batch size $5 \mathrm{~g}$. By using these values of factors, triplicate batches of NS s were prepared. Fig. 3 represents an overlay plot showing the optimized parameters suggested by DoE software (Design-Expert 10.0.3) to get the responses in required range.

\section{Calculation of percentage drug loading}

Drug loading for spray dried and rotary dried nanoparticles was also calculated; for spray dried, it was $74.1 \%$ and for rotary dried nanoparticles it was $50.8 \%$.

\section{Particle size and zeta potential of optimized batch}

Initial particle size of CRM-R was found to be $30 \mu \mathrm{m}$ which was decreased to $760 \mathrm{~nm}$ after conversion into NS (CRM-NS) with polydispersity index (PDI) of 0.445 . When this NS was further solidified in nanoparticles using spray drying and rotary evaporation, the particle size got further reduced. The particle size [PSD d (90)] of CRM-NS (SD) and CRM-NS (RD) was found to be 567 and $660 \mathrm{~nm}$, respectively, with a PDI value of 0.212 and 0.416 . It is important to note that the lower PDI $(<0.5)$ value indicates very good particle size distribution. This was observed for CRM-NS, CRM-NS (SD), and CRM-NS (RD); however, a better distribution was observed for CRM-NS (SD) as compared to CRM-NS and CRM-NS (RD). The zeta potential of CRM-NS, CRM-NS (SD), and CRM-NS (RD) was found to be $-27.29,-28.16$, and $-27.34 \mathrm{mV}$, respectively.

\section{Micromeritic evaluation of powders}

For a dried nanoparticle, to convert it into a suitable drug delivery system, its flow plays major role. Hence, these powders were subjected for evaluation of micromeritic parameters such as angle of repose, bulk and tap density, Hausner's ratio, and Carr's index. It has been observed that spray dried powders (CRM-NS [SD] powders) have shown comparatively better flow as angle of repose value was 9.6 $6^{\circ}$ and compaction properties as Carr's index and Hausner's ratio were 25 and 1.30, respectively, as compared to rotary evaporation method (CRM-NS [RD] powders). The results are shown in Table 4. This could be attributed to recovery of unagglomerated powders from spray dryer with higher surface area as compared to rotary evaporator. Similar observations were recorded when these powders were blended with other excipients that have been used to prepare their tablets (Table 4).

\section{Solubility studies}

The results of solubility studies revealed that aqueous solubility of raw CRM (CRM-R) was $66.7 \mu \mathrm{g} / \mathrm{mL}$, whereas, there was three folds increase in solubility of CRM-NS (RD) after rotary evaporation, i.e., $200 \mu \mathrm{g} / \mathrm{mL}$. The powder obtained by spray drying (CRM-NS [SD]) has shown five folds increase in solubility ( $333 \mu \mathrm{g} / \mathrm{mL}$ ). Hence, conversion of CRM into nanoparticle form has caused reduction in particle size of CRM, which has been decreased further through spray drying and rotary evaporation. This could be the reason behind enhancement in CRM's solubility.

\section{Dissolution studies}

Dissolution study was carried out for raw CRM-R, CRM-NS (RD) and CRM-NS (SD) powders, CRM-R, CRM-NS (RD), and CRM-NS (SD) tablets, respectively. The results are presented in Fig. 4. The data revealed significant increase $(\mathrm{p}<0.05)$ in dissolution rate of CRM-NS $(R D)$ and CRM-NS (SD) powders, CRM-NS (RD), and CRM-NS (SD) tablets as compared to raw CRM (CRM-R) powder and tablets. The drug release from raw CRM powder and tablet was just $22.15 \%$ and $20.16 \%$ in 60 minutes, whereas it was 76.89, 100.16, 72.43, and 99.98\% from CRM-NS (RD) and CRM-NS (SD) powders, CRM-NS (RD) and CRM-NS (SD) tablets, respectively. Moreover, it was observed that more than 90\% drug got released from spray dried CRM nanoparticles in their 


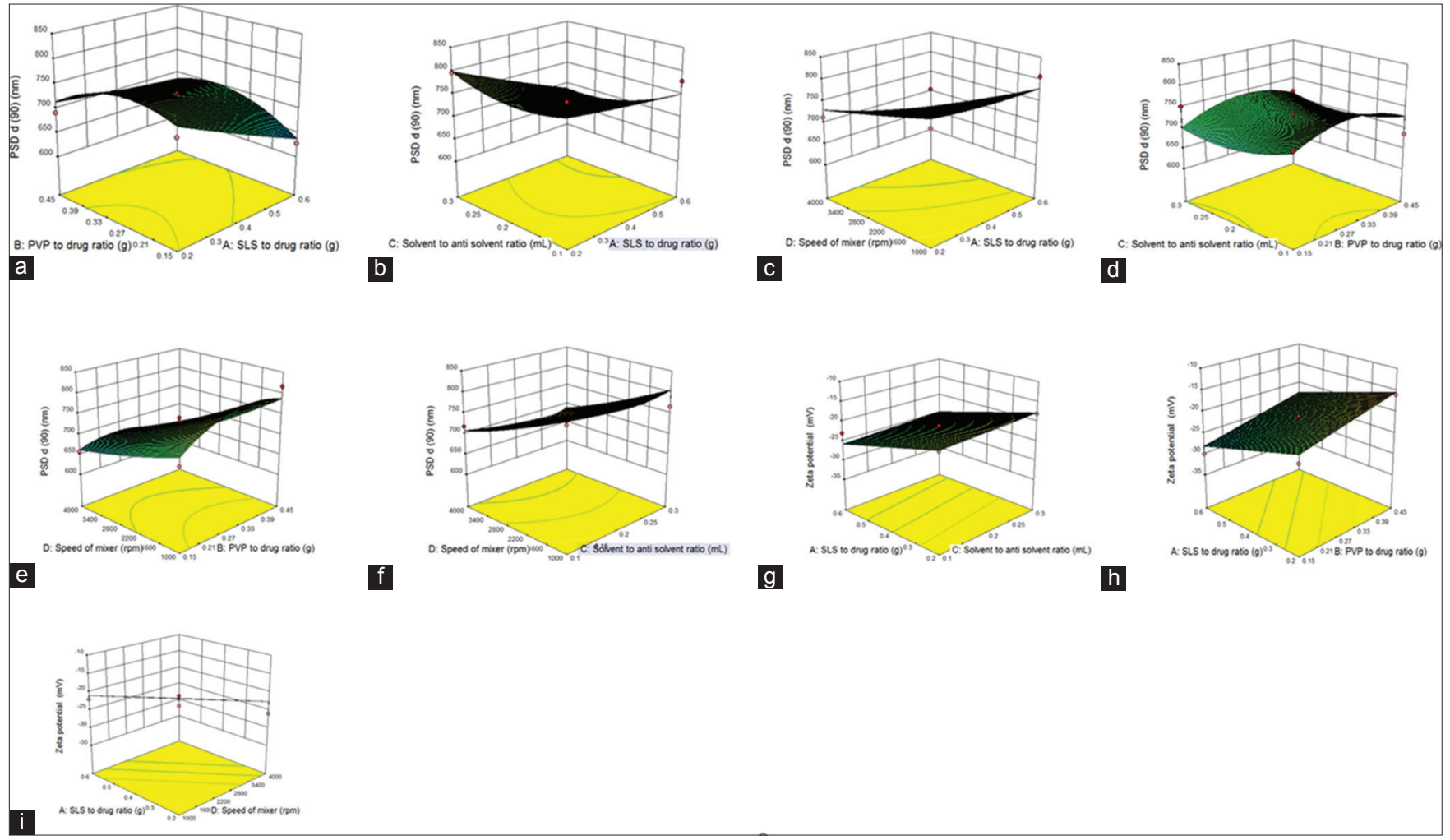

Figure 2: 3D plots for effect of SLS to CRM ratio, PVP K60 to CRM ratio, solvent to antisolvent ratio, and speed of mixing on particle size distribution [i.e. PSD d (90)] (a-f) and zeta potential (g-i)

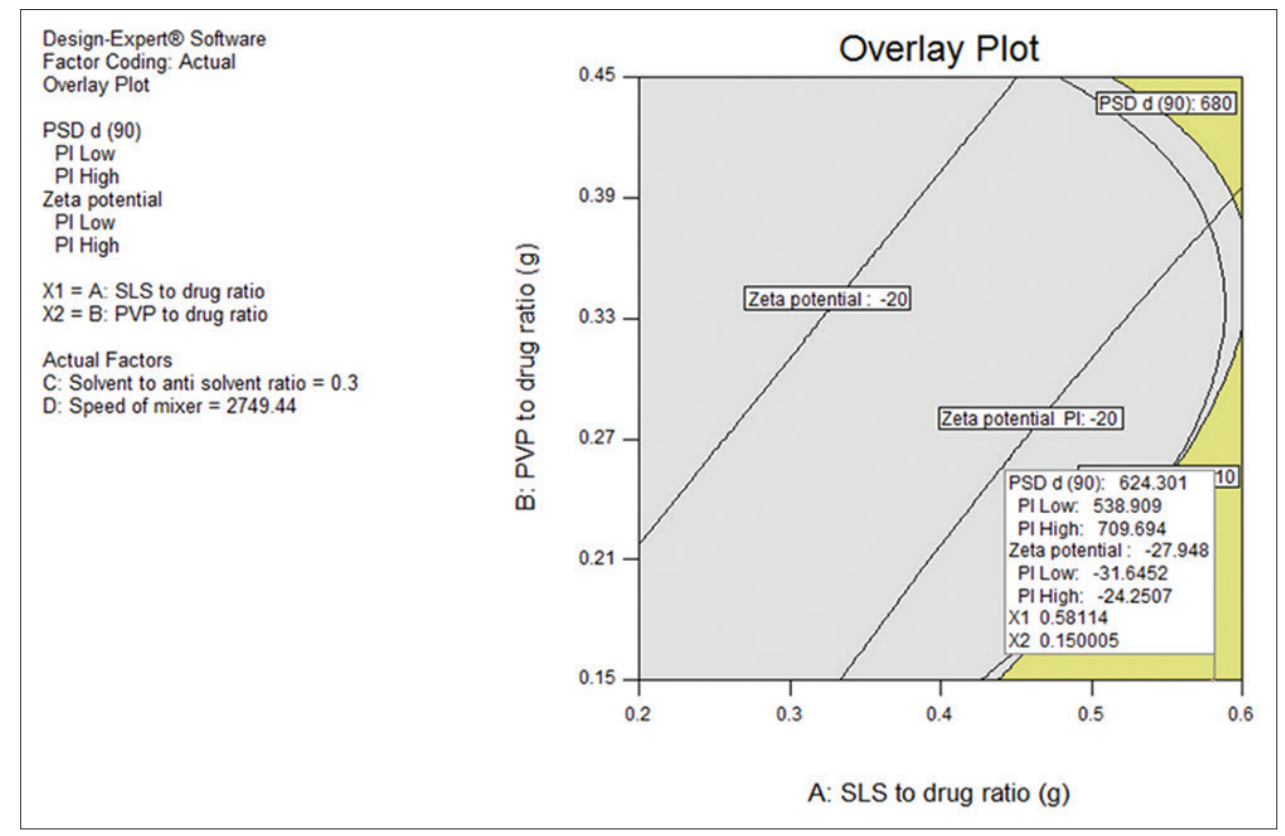

Figure 3: Overlay plot for optimized parameters of CRM nanosuspension

Table 4: Micromeritic characteristics of nanoparticles and tablet blend

\begin{tabular}{|c|c|c|c|c|c|c|}
\hline S.No. & Nanoparticles/tablet blend & $\begin{array}{l}\text { Bulk density } \\
\left(\mathrm{g} / \mathrm{cm}^{3}\right)\end{array}$ & $\begin{array}{l}\text { Tap density } \\
\left(\mathrm{g} / \mathrm{cm}^{3}\right)\end{array}$ & Angle of repose & Hausner's ratio & $\begin{array}{l}\text { Carr's compressibility } \\
\text { index }(\%)\end{array}$ \\
\hline 1 & CRM-NS(RD) powders & 0.42 & 0.56 & 14.8 & 1.33 & 25 \\
\hline 2 & CRM-NS(SD) powders & 0.39 & 0.52 & 9.6 & 1.30 & 25 \\
\hline 3 & CRM-NS(RD) tablet blend & 0.47 & 0.58 & 22.09 & 1.23 & 18.9 \\
\hline 4 & CRM-NS(SD) tablet blend & 0.45 & 0.55 & 18.26 & 1.22 & 18.18 \\
\hline
\end{tabular}




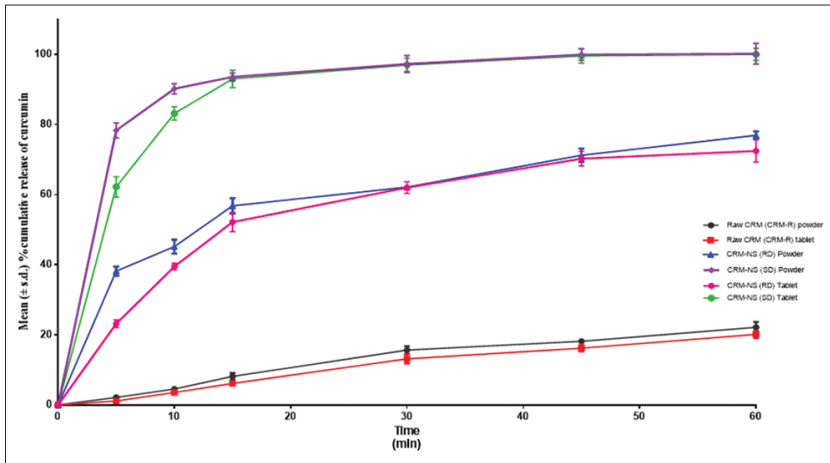

Figure 4: Mean ( \pm SD) \% cumulative release of CRM from Raw CRM (CRM-R) powder and tablet, CRM NS (RD) powder and tablet, and CRM NS (SD) powder and tablet, respectively powder as well as tablet form. The little delay in release from tablet was due to its disintegration. The significant increase in drug release was also observed from rotary dried powders as well as their tablets; however, these were comparatively lesser than that of spray dried powders. This could be attributed to reduced particle size and better micromeritic properties of CRM NS obtained by spray drying, as the particles were free flowing with absence of agglomeration. Particle size reduction was obtained from rotary dried NS also; however, they were having comparatively less flow as that of spray dried products. This could have delayed the wetting of drug in the dissolution medium and thereby delayed dissolution. The increase in drug release from dried nanoparticles as that of CRM-R powder in terms of number of folds is shown below. It is important to note that the observations for CRM-R tablets were similar as that of CRM-R powder.

CRM-NS (RD) tablets (3.27 folds) < CRM-NS (RD) powder (3.47 folds) $<$ CRM-NS (SD) tablets (4.51 folds) < CRM-NS (SD) powder (4.52 folds).

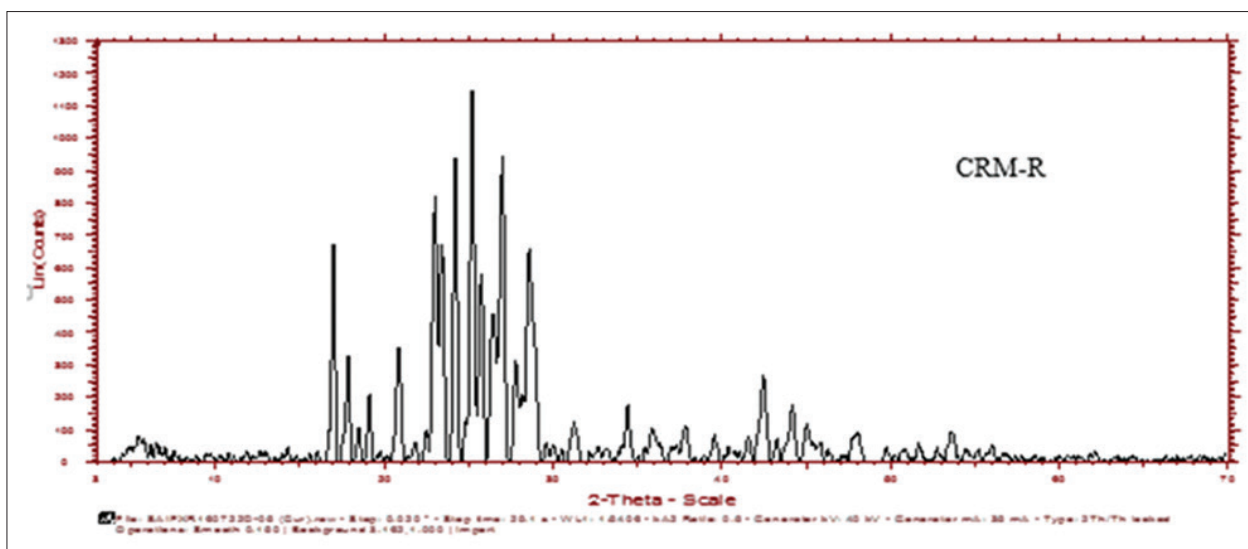

a

$5 b$.

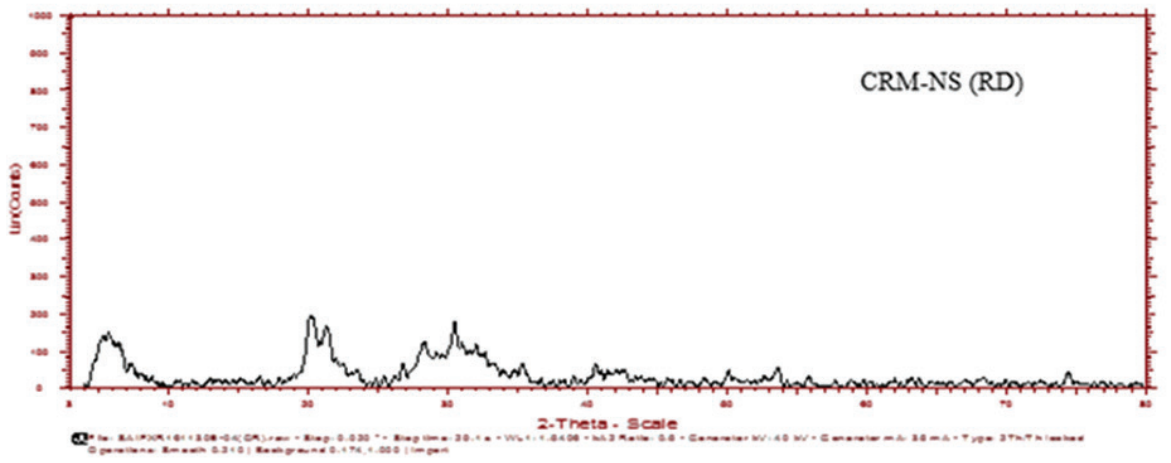

b

$5 c$.

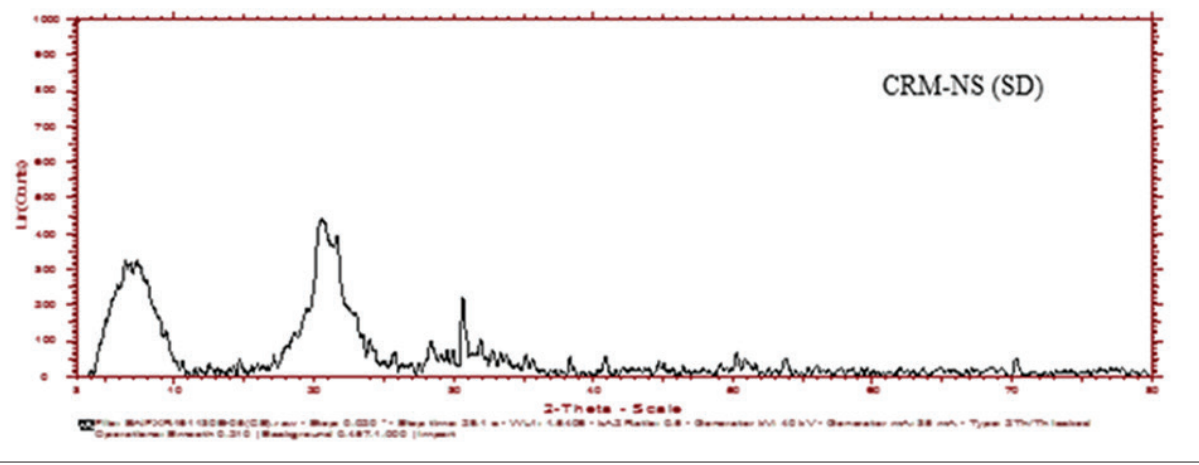

Figure 5: PXRD data for a. CRM-R, b. CRM-NS (RD), and c. CRM-NS (SD) 


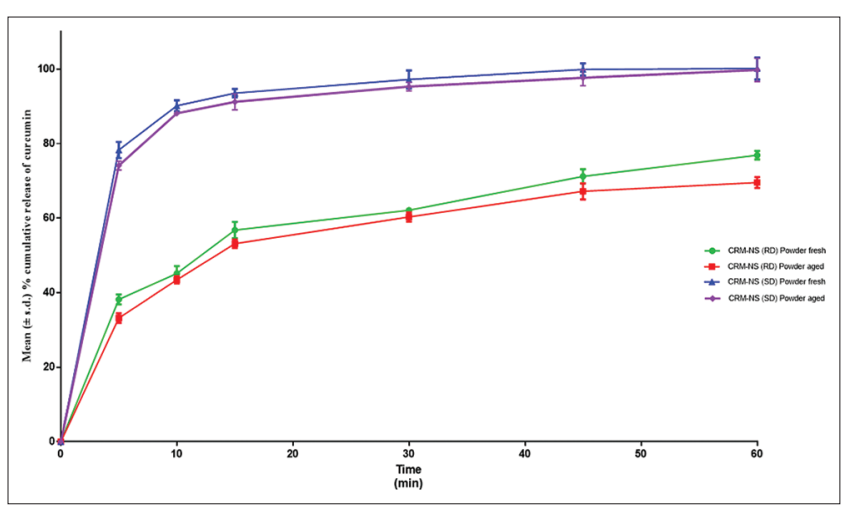

Figure 6: Comparison of dissolution profiles of fresh CRM-NS (SD) and CRM-NS (RD) to that of their corresponding aged formulations that were kept for accelerated stability studies

\section{PXRD analysis}

As shown Fig. 5a, the diffraction pattern of CRM powder (CRM-R) revealed several sharp high-intensity peaks at diffraction angles $(2 \theta)$ of $16.47^{\circ}, 17.82^{\circ}, 17.54^{\circ}, 18.46^{\circ}, 19.08^{\circ}, 20.83^{\circ}, 22.47^{\circ}, 22.98^{\circ}, 23.39^{\circ}$, $24.18^{\circ}, 25.18^{\circ}, 25.72^{\circ}, 26.97^{\circ}, 27.77^{\circ}$, and $34.43^{\circ}$. This suggested that the drug existed as crystalline material. While in case of both, CRMNS-SD and CRM-NS-RD a halo pattern without any peak of drug was observed. This revealed that the nanoparticles prepared by antisolvent method yielded amorphous particles. Hence, it could be inferred that the enhancement in drug dissolution was not only due to a reduction in particle size but also due to generation of amorphous form [34].

\section{Stability studies}

The accelerated stability study was carried out for CRM-NS (SD) and CRM-NS (RD) powders at $40^{\circ} \mathrm{C}$ and $75 \% \mathrm{RH}$ for 6 months. The results revealed no significant difference ( $p>0.05)$ in angle of repose values of fresh and aged CRM-NS (SD) and CRM-NS (RD) powders. The initial value of angle of repose was 9.6 and $14.8^{\circ}$ for fresh CRM-NS (SD) and CRM-NS (RD), respectively. On completion of 6 months stability studies, these values were $10.23^{\circ}$ and $16.62^{\circ}$ for aged CRM-NS (SD) and CRM-NS (RD), respectively. In case of particle size, a significant increase $(\mathrm{p}<0.05)$ was observed in case of CRM-NS (RD). Initially, the particle size of CRM-NS (RD) was $660 \mathrm{~nm}$ which increased to $910 \mathrm{~nm}$ in 6 months under accelerated conditions. This increase in particle size was about 1.38 folds. However, there is no such significant increase was observed for aged CRM-NS (SD) particles as compared to fresh one. Initial particle size of fresh CRM-NS (SD) was found to be $567 \mathrm{~nm}$ and aged was found to be $602 \mathrm{~nm}$. Further, the dissolution study of fresh and aged nanoparticles revealed significant decrease (f2 $=48.84$ which is $<50$ ) in the dissolution profile of aged CRM-NS (RD) as compared to fresh CRM-NS (RD) (Fig. 6). This decrease in dissolution profile of aged CRM-NS (RD) could be attributed to its increased particle during storage at accelerated stability conditions. Whereas, in case of spray dried nanoparticles such changes were not observed. The f2 value between dissolution profiles of aged and fresh CRM-NS (SD) powders was 60.89 , i.e., above 50 , which reveals acceptably similar dissolution profile (Fig. 6).

\section{CONCLUSION}

In this study, CRM NS was formulated by the application of solventantisolvent precipitation technique. Based on BBD, total 25 experiments were performed by varying four critical formulation and processing parameters that can affect their particle size [PSD d (90)] and zeta potential. These were SLS to CRM ratio, PVPK-60 to CRM ratio, speed of mixing and S/AS ratio. The optimized formulation was further solidified into free flowing powder using spray drying and rotary evaporation techniques. Drying of NS further caused reduction in the particle size with good particle size distribution [PSD d (90)]. XRD results revealed that the formed nanoparticles were amorphous in nature. The data revealed that spray dried nanoparticles of CRM were found superior to that of rotary dried nanoparticles in terms of particle size [PSD d (90)], solubility, dissolution and powder flow. Furthermore, when the nanoparticles dried by using two techniques were subjected for accelerated stability studies, it was observed that spray dried nanoparticles were able to retain their particles size, flow as well as dissolution behavior. Hence, it was concluded that spray dried CRM nanoparticles could forecast a better scope of scale up and commercialization.

\section{ACKNOWLEDGMENTS}

Authors would like to express their thanks Ms. Paranjeet Kaur, Junior Research Fellow, School of Pharmaceutical Sciences, Lovely Professional University, for providing necessary support.

\section{REFERENCES}

1. Priyadarsini KI. The chemistry of curcumin: From extraction to therapeutic agent. Molecules 2014;19(12):20091-112.

2. Sharma RA, McLelland HR, Hill KA, Ireson CR, Euden SA, Manson MM, et al. Pharmacodynamic and pharmacokinetic study of oral Curcuma extract in patients with colorectal cancer. Clin Cancer Res 2001;7(7):1894-900.

3. Sharma OP. Antioxidant activity of curcumin and related compounds. Biochem Pharmacol 1976;25(15):1811-2.

4. Negi PS, Jayaprakasha GK, Jagan Mohan Rao L, Sakariah KK. Antibacterial activity of turmeric oil: A byproduct from curcumin manufacture. J Agric Food Chem 1999;47(10):4297-300.

5. Apisariyakul A, Vanittanakom N, Buddhasukh D. Antifungal activity of turmeric oil extracted from Curcuma longa (Zingiberaceae). J Ethnopharmacol 1995;49(3):163-9.

6. Itthipanichpong C, Ruangrungsi N, Kemsri W, Sawasdipanich A. Antispasmodic effects of curcuminoids on isolated guinea-pig ileum and rat uterus. J Med Assoc Thai 2003;86 Suppl 2:S299-309.

7. Dhar ML, Dhar MM, Dhawan BN, Mehrotra BN, Ray C. Screening of Indian plants for biological activity: I. Indian J Exp Biol 1968;6(4):232-47

8. Mazumder A, Raghavan K, Weinstein J, Kohn KW, Pommier Y. Inhibition of human immunodeficiency virus Type-1 integrase by curcumin. Biochem Pharmacol 1995;49(8):1165-70.

9. Aggarwal BB, Harikumar KB. Potential therapeutic effects of curcumin, the anti-inflammatory agent, against neurodegenerative, cardiovascular, pulmonary, metabolic, autoimmune and neoplastic diseases. Int J Biochem Cell Biol 2009;41(1):40-59.

10. Mohanty C, Das M, Sahoo SK. Emerging role of nanocarriers to increase the solubility and bioavailability of curcumin. Expert Opin Drug Deliv 2012;9(11):1347-64

11. Anand P, Kunnumakkara AB, Newman RA, Aggarwal BB. Bioavailability of curcumin: Problems and promises. Mol Pharm 2007;4(6):807-18.

12. Shen L, Ji HF. The pharmacology of curcumin: Is it the degradation products? Trends Mol Med 2012;18(3):138-44.

13. Tiyaboonchai W, Tungpradit W, Plianbangchang P. Formulation and characterization of curcuminoids loaded solid lipid nanoparticles. Int $\mathbf{J}$ Pharm 2007;337:299-306.

14. Maiti K, Mukherjee K, Gantait A, Saha BP, Mukherjee PK. Curcuminphospholipid complex: Preparation, therapeutic evaluation and pharmacokinetic study in rats. Int J Pharm 2007;330:155-63.

15. Shoba G, Joy D, Joseph T, Majeed M, Rajendran R, Srinivas PS. Influence of piperine on the pharmacokinetics of curcumin in animals and human volunteers. Planta Med 1998;64(4):353-6.

16. Mishra S, Narain U, Mishra R, Misra K. Design, development and synthesis of mixed bioconjugates of acid-glycine, curcumin-glycine/ alanine and curcumin-glycine-piperic acid and their antibacterial and antifungal properties. Bioorg Med Chem 2005;13(8):1477-86.

17. Available from: http://www.dolcas-biotech.com/products/bcm $95 /$. [Last accessed on 2016 Dec 17].

18. John VD, Kuttan G, Krishnankutty K. Anti-tumour studies of metal chelates of synthetic curcuminoids. J Exp Clin Cancer Res $2002 ; 21(2): 219-4$

19. Sui Z, Salto R, Li J, Craik C, Ortiz de Montellano PR. Inhibition of the HIV-1 and HIV-2 proteases by curcumin and curcumin boron complexes. Bioorg Med Chem 1993;1(6):415-22.

20. Yallapu MM, Jaggi M, Chauhan SC. Curcumin nanomedicine: A road to cancer therapeutics. Curr Pharm Des 2013;19(11):1994-2010. 
21. Muller RH, Cornelia MK. Drug nanocrystals of poorly soluble drugs produced by high pressure homogenization. Int $\mathrm{J}$ Pharm 2006;62(1):3-16

22. Singh SK, Vaidya Y, Gulati M, Bhattacharya S, Garg V, Pandey NK. Nanosuspension: Principles, perspectives and practices. Curr Drug Deliv 2016;13(8):1222-46.

23. Patravale VB, Date AA, Kulkarni RM. Nanosuspensions: A promising drug delivery strategy. J Pharm Pharmacol 2004;56(7):827-40.

24. Verma S, Gokhale R, Burgess DJ. A comparative study of top-down and bottom-up approaches for the preparation of micro/nanosuspensions. Int J Pharm 2009;380:216-2.

25. Cal K, Sollohub K. Spray drying technique. I: Hardware and process parameters. J Pharm Sci 2010;99(2):575-86.

26. Schmid K, Arpagaus C, Friess W. Evaluation of the nano spray dryer B-90 for pharmaceutical applications. Pharm Dev Technol 2011;16(4):287-94.

27. Martin A, Bustamante P, Chun AH. In: Sinko PJ, editor. Physical Pharmacy. USA: Lippincott Williams \& Wilkins; 2011.

28. Masters K. Spray Drying, an Introduction to Principles of
Operational Practice and Applications. $2^{\text {nd }}$ ed. London: Leonard Hill Books; 1976

29. Beg S, Katare OP, Saini S, Garg B, Khurana RK, Singh B. Solid selfnanoemulsifying systems of olmesartan medoxomil: Formulation development, micromeritic characterization, in vitro and in vivo evaluation. Powder Technol 2016;294:93-104.

30. Kaur P, Singh SK, Garg V, Gulati M, Vaidya Y. Optimization of spray drying process for formulation of solid dispersion containing polypeptide-k powder through quality by design approach. Powder Technol 2005;284:1-11.

31. Shah VP, Tsong Y, Sathe P, Liu JP. In vitro dissolution profile comparison-statistics and analysis of the similarity factor, f2. Pharm Res 1998;15(6):889-96.

32. Mahesh KV, Singh SK, Gulati M. A comparative study of top-down and bottom-up approaches for the preparation of nanosuspensions of glipizide. Powder Technol 2014;256:436-9.

33. Shah SR, Parikh RH, Chavda JR, Sheth NR. Application of plackettburman screening design for preparing glibenclamide nanoparticles for dissolution enhancement. Powder Technol 2013;235:405-11. 